\title{
Maximiser les apprentissages : trois leçons tirées de la psychopédagogie
}

\author{
Maximizing learning: Three lessons from educational psychology
}

\section{Georges BORDAGE}

Department of Medical Education, College of Medicine, University of Illinois at Chicago, USA

Manuscrit reçu le 7 octobre 2016 ; commentaires éditoriaux formulés à l'auteur le 2 novembre 2016 ; accepté pour publication le 9 novembre 2016

\author{
Mots-clés \\ stratégies pédago- \\ giques ; psychopéda- \\ gogie ; acquisition des \\ connaissances ; \\ transfert des connais- \\ sances ; pratique \\ mixte ; évaluation \\ pour apprendre.
}

\section{Keywords} instructional strategies; educational psychology; knowledge acquisition; mixed practice; knowledge transfer; assessment for learning
Résumé-Contexte et problématique : Nous utilisons souvent dans nos programmes d'enseignement des stratégies pédagogiques moins performantes parce que nous ne prenons pas en compte des données probantes de la psychopédagogie. Exégèse : Suite à une analyse approfondie de la littérature, Rohrer et Pashler recommandent trois stratégies pour maximiser l'apprentissage: étaler les contenus dans le temps, faire pratiquer de façon mixte l'application des connaissances et vérifier ses connaissances pour apprendre.

Abstract - Background: We often use educational strategies in medical education that are less than optimal had we been guided by evidence from educational psychology. Findings: In an extensive analysis of the literature, Rohrer and Pashler recommend three strategies to maximize learning: spacing content over time, mixed practice, and learning through testing.

\section{Problématique}

Étudiants et enseignants en médecine, et en sciences de la santé en général, sont toujours à l'affut de nouvelles approches susceptibles d'améliorer leurs habitudes d'apprentissage, leurs méthodes d'enseignement et leurs programmes de formation. En 2010, Rohrer et Pashler ${ }^{[1]}$ ont mené une vaste analyse des facteurs favorisant l'apprentissage chez les humains. Ils ont pu ainsi identifier trois facteurs principaux. Les deux premiers ont trait à des stratégies d'enseignement et s'adressent donc aux

Article commenté : Rohrer D \& Pashler H. Recent Research on Human Learning Challenges Conventional Instructional Strategies. Educ Res 2010;38:406-12 
enseignants : 1) étaler les contenus dans le temps et 2) faire pratiquer de façon mixte l'application des connaissances. Le troisième facteur concerne une stratégie d'apprentissage et s'adresse donc aux étudiants : 3 ) vérifier ses connaissances pour apprendre. Bien que ces facteurs reposent sur de solides données probantes, ils sont souvent négligés dans le cadre des pratiques courantes en éducation. À partir de chacun de ces facteurs, je vais faire un parallèle entre les pratiques courantes et ce qui pourrait être fait pour améliorer les apprentissages tout en donnant quelques exemples tirés de recherches en pédagogie médicale.

\section{Étaler les contenus}

Malgré diverses réformes, les programmes de formation en médecine sont encore souvent organisés en une succession de cours distincts, condensés et isolés les uns des autres; en d'autres termes, des silos de disciplines ou de systèmes d'organe. Or les données probantes de Rohrer et Pashler indiquent que les étudiants ont une meilleure rétention à long terme de leurs connaissances lorsque les contenus sont présentés en plus petites quantités sur une période de temps modérément longue ${ }^{[1]}$. L'étudiant peut ainsi réactiver périodiquement ses connaissances antérieures et continuellement bâtir sur celles-ci, au fur et à mesure que le programme se déroule. Par exemple, Raman et collaborateurs ${ }^{[2]}$ ont démontré que la rétention a augmenté de $150 \%$ (taille de l'effet $=1.46$ ) trois mois après un cours à des résidents sur l'évaluation et le maintien nutritionnel lorsque les contenus furent présentés, non pas en une seule séance de quatre heures, c'est-à-dire un type d'enseignement couramment utilisé, mais plutôt en quatre portions d'une heure sur quatre semaines. Rohrer et Pashler recommandent que « ...si vous voulez retenir l'information sur une longue période de temps, l'intervalle de temps pris pour apprendre devrait être modérément long ${ }^{[1]}$.

Nous avons l'habitude en médecine de présenter nos contenus sur un mode «bolus ». Une meilleure stratégie consisterait à structurer nos programmes sur un mode "soluté », en spirale à la Bruner ${ }^{[3]}$, une forme particulière d'étalement, où les étudiants peuvent revisiter, réactiver leurs connaissances et constamment bâtir sur celles-ci. Par exemple, les divers contenus de l'appareil cardio-vasculaire ont été étalés et revus sur 10 semestres dans le programme de médecine en spirale mis en place à La Charité (Université de Berlin) ${ }^{[4]}$; voir figure 1.

\section{Pratiquer de façon mixte}

Aujourd'hui encore, les enseignants offrent souvent peu d'occasions à leurs étudiants de mettre en pratique leurs nouvelles connaissances. Le plus souvent, on se contente d'exercices de fin de chapitre en lien direct avec les contenus qui viennent d'être enseignés. Ce type de pratique dit « en bloc » consiste à mettre en pratique des mêmes habiletés en les répétant. Or les données probantes ${ }^{[1]}$ démontrent que le fait de pratiquer de façon « mixte » plutôt qu'en bloc, c'est-à-dire en sollicitant plusieurs types d'habiletés en même temps, améliore davantage l'apprentissage. Par exemple, Hatala et collaborateurs ${ }^{[6]}$ ont démontré qu'apprendre la lecture de l'électrocardiogramme selon un mode de pratique mixte, soit en considérant trois types de pathologies à la fois, améliore l'apprentissage de $53 \%$ (taille de l'effet = 1.6) comparativement à l'exercice sur une seule pathologie à la fois, de façon séquentielle.

La pratique mixte facilite le transfert des connaissances en forçant les étudiants à comparer et à contraster différentes solutions, et à choisir celle qui est la plus appropriée compte tenu du problème à résoudre, ce que Rohrer et Pashler nomment leur capacité à discriminer ${ }^{[1]}$. Par exemple, il vaut mieux évaluer une douleur thoracique en considérant plusieurs systèmes simultanément (par exemple : cardiaque, respiratoire et musculo-squelettique) plutôt qu'un seul système à la fois, comme c'est souvent le cas dans les programmes organisés en système d'organe. Cette stratégie rappelle la notion de "pratique délibérée » d'Ericsson $^{[7]}$, qui consiste à offrir aux étudiants, de façon délibérée, plusieurs occasions de pratiquer pendant et après les cours pour faciliter l'acquisition et le transfert des connaissances. Pour maximiser les transferts à de nouvelles situations (transferts dits lointains), il importe aussi de pratiquer en mettant en jeu à la fois plusieurs concepts dans plusieurs contextes ${ }^{[8]}$. 


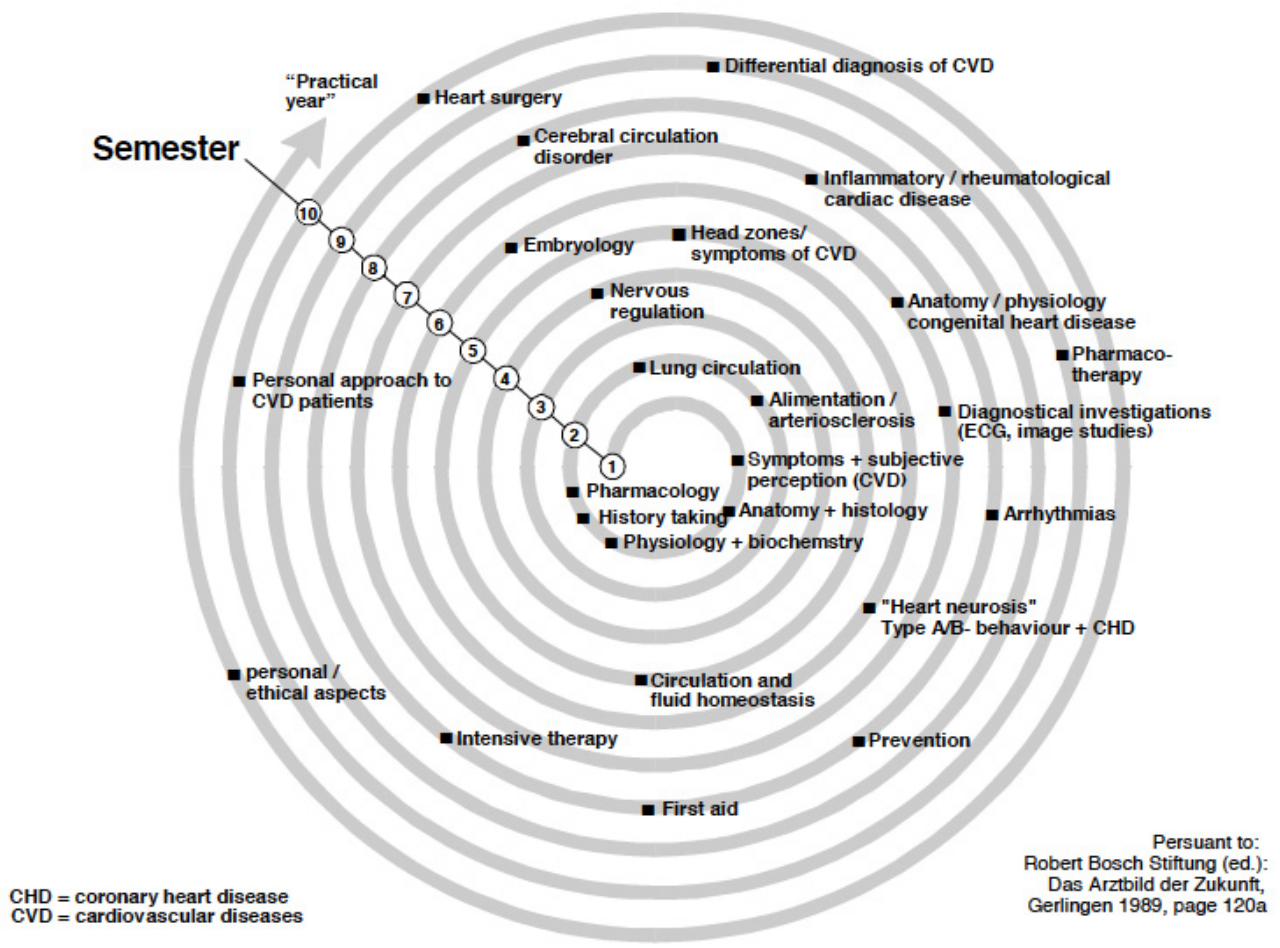

Fig. 1. Exemple d'un programme en spirale pour l'appareil cardio-vasculaire, Faculté de médecine à La Charité (Université de Berlin) - Reproduit avec permission ${ }^{[5]}$.

\section{S'évaluer pour apprendre}

La stratégie habituelle des étudiants pour préparer les examens est de revoir leurs notes de cours ou d'anciennes questions d'examen. Ici encore, les données probantes indiquent que les étudiants gagneraient davantage en consacrant la même quantité de temps à vérifier leurs connaissances plutôt que de relire leurs notes ou des vieilles questions ${ }^{[1]}$. Larsen et collaborateurs ${ }^{[9]}$ ont observé une augmentation de $50 \%$ de la rétention des connaissances (taille de l'effet $=0.68$ ) concernant le traitement d'un état de mal convulsif et le diagnostic de la myasthénie grave, six mois plus tard, lorsque des résidents se sont livrés à des examens formatifs avec rétroaction, comparativement à ce qui est observé lorsqu'ils utilisent la même quantité de temps pour revoir leurs notes de cours. Ainsi, Rohrer et Pashler recommandent de «...vérifier ses connaissances parce que cela est plus efficace que de passer la même quantité de temps à relire ses notes ${ }^{[1]}$. De plus, les données probantes indiquent que « construire une réponse (par exemple à une question à réponse ouverte et courte -QROC) consolide davantage les connaissances en mémoire que des questions à choix de réponses (QCR), même si l'examen final ne contient que des QCR » ${ }^{[1]}$. Cette forme constructive de questions force le retrait d'information en mémoire plutôt que la simple reconnaissance. Le défi pour les enseignants est de préparer des examens formatifs nécessitant l'élaboration de réponses et d'offrir une rétroaction immédiate. Une autre stratégie d'emploi de ce type d'évaluation formative consiste à utiliser des logiciels de réactivation des connaissances comme Anki, Mnémosyne et SuperMemo ${ }^{[10]}$.

En résumé, trois défis sont à relever.

- Les enseignants devraient:1) structurer les programmes en spirale afin d'offrir aux étudiants de 
multiples occasions de revoir et constamment augmenter et consolider leur bagage de connaissances et 2) les faire pratiquer, beaucoup, de façon délibérée et mixte afin de faciliter l'intégration et le transfert des connaissances.

- Quant aux étudiants, ils devraient : 3) vérifier leurs connaissances de façon formative pour mieux apprendre.

Pourquoi des pratiques moins performantes sontelles si populaires ? Ce n'est pas par manque de preuve, avec des tailles d'effet importantes (en l'occurrence : $0.68,1.46,1.6)$. Selon Rohrer et Pashler, le statu quo est maintenu parce que les enseignants ont des croyances (erronées) selon lesquelles ces nouvelles stratégies sont moins efficaces que leurs pratiques courantes, parce qu'elles produisent, entre autre, davantage d'erreurs pendant l'apprentissage. Il est plus facile de maintenir ses croyances pour ne pas changer. Changer exige que les enseignants et les étudiants se débarrassent de leurs croyances erronées, restructurent leur conception mentale de l'apprentissage et acceptent de participer à des activités de perfectionnement pédagogique. Mais encore faut-il qu'ils reconnaissent l'importance de le faire !

\section{Références}

1. Rohrer D, Pashler H. Recent Research on Human Learning Challenges Conventional Instructional Strategies. Educ Res 2010;38:406-12.

2. Raman M, Mclaughlin K, Violato C., Rostom A, Allard JP, Coderre S. Teaching in small portions dispersed over time enhances long-term knowledge retention. Med Teach 2010;32:250-5.

3. Bruner, JS. The Process of Education. Cambridge, MA: Harvard University Press, 1960.
4. Burger, W. Der Reformstudiengang Medizin an der Charité. Bundesgesunheltsbl-GesundheltsforschGesundheltsschutz, 2006;49:337-43.

5. Groupe de travail pour la réforme du programme de médecine, Faculté de médecine à La Charité, Université de Berlin. Traduit de: Das Arztbild der Zukunft: Analysen künftiger Anforderungen an den Arzt; Konsequenzen für die Ausbildung und Wege zu ihrer Reform / Arbeitskreis Medizinerausbildung. RobertBosch-Stiftung. Gerlingen: Bleicher, 1989, 120a.

6. Hatala RM, Brooks LR, Norman GR. Practice makes perfect: the critical role of mixed practice in the acquisition of ECG interpretation skills. Adv Health Sci Educ Theory Pract 2003;8:17-26.

7. Ericsson E. Acquisition and maintenance of medical expertise: A perspective from the expert-performance approach with deliberate practice. Acad Med 2004;79:S70-S81.

8. Kulasegaram K, Min C, Howey E, Neville A, Woods N, Dore K, Norman G. The mediating effect of context variation in mixed practice for transfer of basic science. Adv Health Sci Educ Theory Pract 2015;20:953-68.

9. Larsen DP, Butler AC, Roediger III HL. Repeated testing improves long term retention relative to repeated study: a randomized controlled trial. Med Educ 2009;43:1174-81.

10. Lineberry M. Memory strength prediction accuracy in learner-adaptive software for application of the spacing and testing effects. American Educational Research Association Annual Meeting, Philadelphia, USA, Avril 2014.

Correspondance et offprints : Georges Bordage, College of Medicine, University of Illinois at Chicago, $808 \mathrm{~S}$ Wood, Chicago, IL 60612-7309, USA.

Mailto: bordage@uic.edu. 